\title{
Correction to: Serum 25-hydroxyvitamin D might be negatively associated with hyperuricemia in U.S. adults: an analysis of the National Health and Nutrition Examination Survey 2007-2014
}

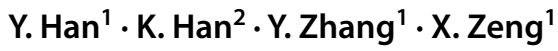

Published online: 3 February 2022

(c) Italian Society of Endocrinology (SIE) 2022
81901667) and CAMS Innovation Fund for Medical Sciences CIFMS 2020-I2M-2-009), the Clinical and translational medical research fund of Chinese Academy of Medical Sciences (no. 2019XK320013).

The original article has been corrected.

Publisher's Note Springer Nature remains neutral with regard to jurisdictional claims in published maps and institutional affiliations.

1 Department of General Internal Medicine, Peking Union Medical College Hospital, Chinese Academy of Medical Sciences, No. 1 Shuaifuyuan, Dongcheng District, Beijing 100730, China

2 Department of Critical Care Medicine, The Affiliated Yantai Yuhuangding Hospital of Qingdao University, Yantai 264000, Shandong, China 